\title{
Understanding and Beliefs about the Etiology and Treatment of Mental Illness in Sodo
}

\section{Introduction}

- Beliefs about and treatment of mental illness are expressed differently among cultures and may impact treatment seeking behaviors ${ }^{1,2,3}$

- Understanding these variables is a critical step in developing culturallyrelevant treatment options ${ }^{4}$

- Current efforts in Ethiopia to address mental illness often lack psychotherapeutic interventions, and assessment of the acceptability of therapy in this context is needed

- This qualitative study aimed to identify clinically and culturally relevant characteristics of the population in Sodo District, Ethiopia to better understand beliefs about the causes of mental illness and perceptions of treatment

\section{Methods}

- Purposeful sampling was used to recruit 48 patients, caregivers, health care providers, and leaders

- Semi-structured, in-depth interviews were conducted, recorded, and transcribed in Amharic and translated into English

- Four coders conducted thematic analysis using NVIVO 12

\section{Discussion}

- Efforts to adapt and integrate psychotherapeutic interventions seem well-aligned with norms and desire for treatment

- Community familiarity with related concepts, such as advice giving, many prove to be a facilitator to implementation of such programs

- These findings suggest possible mechanisms of improving treatment options to support diminishing the global treatment gap

\section{References}

1. Eaton J, Agomoh A. Developing mental health services in Nigeria. Soc Psychiatry Psychiatr Epidemiol. 2008;43(7):552-58.

Mulatu M. Perceptions of mental and physical illnesses in north-western Ethiopia $J$ Health Psychol. 1999:4(4):531-49.

Mendenhall E, De Silva M, Hanlon C, Petersen I, Shidhaye R, Jordans M, et al. Acceptability and feasibility of using non-specialist health workers to deliver mental health care: stakeholder perceptions from the PRIME district sites in Ethiopia, India, Nepal, South Africa, and Uganda. Soc Sci Med. 2014;118:33-42.

Divan G. 'From there to here': adapting child and adolescent mental health interventions for low-resource settings. J Child Psychol Psychiatry. 2017;58(3):325-27.

\section{Results}

- Results from this study indicate that mental illness is understood to stem from spiritual attacks (i.e., evil doings of other people), stress (e.g., challenging life events), and/or trauma

- Existing methods of coping include relying on church/religious beliefs, seeking advice, drinking alcohol, sleeping, socializing, walking, or seeking justice (i.e., using someone as a mediator to make peace)

- Informal methods of treatment include using holy water or seeking advice from community leaders

- Patients and providers overwhelmingly noted that counseling and seeking advice were appropriate methods to seek relief from mental health symptoms

\begin{tabular}{c|c|c} 
Themes & Selected Examples \\
\hline & "We saw unexpected individuals getting better, but what is not
\end{tabular}

Etiology $\quad$ Spiritual Attacks

Stress

Trauma

Current methods: church/

Treatment Beliefs drinking alcohol, sleeping, socializing, walking, seeking justice

Informal treatment: holy water or seeking advice from community leaders/elders seeking advice
"We saw unexpected individuals getting better, but what is not believe that it is related to spiritual doings in the household." "When I think about my life, I feel stressed and shocked. I am day laborer and I don't know what to feed my children and $m$ family... when I see the suffering of my family and my children I wish to die."

"My father's sister got mentally ill suddenly because of her child's death, so now we should not talk about her child."

Question: "In this society if someone is in some sort of problem, where do they prefer to go to get help?"

Answer: "In our society, advice is the first thing... they will go to the nearest person and take advice if possible."

"The health facilities need to work in collaboration and in a close relation with them. For example, religious institutions had a big role regarding the prevention of HIV, they had contributed a lot...those people will go to a holy water. People might go anywhere they want depending on their religious belief. In addition to that, they also took the medication as far as it's necessary. Thus, I believe that both can work together." "I told you what I have in mind and you will advise me things so that I will get calm. I just told you what I feel, and you advise me a good advice...obviously, you don't advise me a bad thing, right? Since you are talking to me patiently, I have also told you what my personal issues. I will be getting back home feeling relaxed because I talk to you about my personal issues." 